\title{
A note on Generalized Operator Quasicontractions in Cone Metric Spaces
}

\author{
S. Radenović, M. Pavlović, Lj. Paunović, Z. Kadelburg
}

\begin{abstract}
In a recent paper [Fixed point theorem for generalized operator quasi-contractive mappings in cone metric spaces, Afrika Mat., DOI 10.1007/ s13370-012-0105-7], X. Zhang has used bounded positive definite linear operators on the given Banach space in an attempt to obtain a more general fixed point result in a normal cone metric space. We will show in this paper that most of the conditions in his theorem are superfluous and that the proof can be obtained in a much easier way, by reducing it to a well known Ćirićs result on quasicontractions in standard metric spaces.
\end{abstract}

Keywords: cone metric space, normal-cone, quasicontraction

\section{Introduction}

L.G. Huang and X. Zhang introduced cone metric spaces in [6], replacing the set of real numbers by an ordered Banach space as the codomain of a metric. Thus, they reconsidered the notion of $K$-metric spaces that was used earlier (see, e.g., [12]). A lot of known metric fixed point and common fixed point results were subsequently extended to this new setting (for a review of these results till 2010 see [7]).

In a recent paper [13], X. Zhang has used bounded positive definite linear operators on the given Banach space in an attempt to obtain a more general fixed point result in a normal cone metric space. We will show in this note that most of the conditions in his theorem are superfluous and that the proof can be obtained in a much easier way, by reducing it to a well known Ćirić's ([1]) result on quasicontractions in standard metric spaces.

\section{Preliminaries}

Manuscript received June 19, 2017 ; accepted October 04, 2017.

S. Radenović is with the University of Belgrade, Faculty of Mechanical Engineering, Beograd, Serbia; M. Pavlović is with the Faculty of Science, University of Kragujevac, Kragujevac, Serbia; Lj. Paunović is with the University of Pristina-Kosovska Mitrovica, Faculty of Teacher Training Prizren-Leposavic, Serbia; Z. Kadelburg is with the University of Belgrade, Faculty of Mathematics, Beograd, Serbia 
We recall some properties of cones and cone metric spaces. The details and proofs can be found in $[6,7]$.

Let $E$ be a real Banach space with the zero vector $\theta$. A proper nonempty and closed subset $K$ of $E$ is called a (convex) cone if $K+K \subset K, \lambda K \subset K$ for $\lambda \geqslant 0$ and $K \cap(-K)=$ $\{\theta\}$. We shall always assume that the cone $K$ has a nonempty interior int $K$ (such cones are called solid).

Each cone $K$ induces a partial order $\preceq$ on $E$ by $x \preceq y \Leftrightarrow y-x \in K . x \prec y$ will stand for $x \preceq y$ and $x \neq y$, while $x \ll y$ will stand for $y-x \in \operatorname{int} K$. The pair $(E, K)$ is an ordered Banach space.

Definition $2.1[2]$ A cone $K$ in a Banach space $(E,\|\cdot\|)$ is called

$1^{\circ}$ normal if

$$
\inf \{\|x+y\|: x, y \in K,\|x\|=\|y\|=1\}>0 ;
$$

$2^{\circ}$ semi-monotone if there exists $k>0$ such that, for all $x, y \in E$,

$$
\theta \preceq x \preceq y \text { implies }\|x\| \leqslant k\|y\| ;
$$

$3^{\circ}$ monotone if, for all $x, y \in E$,

$$
\theta \preceq x \preceq y \text { implies }\|x\| \leqslant\|y\|,
$$

i.e. it is semi-monotone with $k=1$.

The next lemma contains results on cones in ordered Banach spaces that are rather old (1940, see [8]). It is interesting that most of the authors (working with normal cones after 2007) do not use these results, which can be applied to reduce a lot of results to the setting of ordinary metric spaces.

Lemma 2.1 $[8,2]$ The following conditions are equivalent for a cone $K$ in the Banach $\operatorname{space}(E,\|\cdot\|)$ :

$1^{\circ} \mathrm{K}$ is normal;

$2^{\circ}$ for arbitrary sequences $\left\{x_{n}\right\},\left\{y_{n}\right\},\left\{z_{n}\right\}$ in $E$,

$$
(\forall n) x_{n} \preceq y_{n} \preceq z_{n} \text { and } \lim _{n \rightarrow \infty} x_{n}=\lim _{n \rightarrow \infty} z_{n}=x \text { imply } \lim _{n \rightarrow \infty} y_{n}=x ;
$$

$3^{\circ} \mathrm{K}$ is semi-monotone;

$4^{\circ}$ there exists a norm $\|\cdot\|_{1}$ on $E$, equivalent to the given norm $\|\cdot\|$, such that the cone $K$ is monotone w.r.t. $\|\cdot\|_{1}$.

The smallest constant $k$ satisfying the inequality (1) is called the normal constant of $K$. It is clear that it is always $k \geqslant 1$. 
Example 2.1 [11] Let $E=C_{\mathbb{R}}^{1}[0,1]$, with $\|x\|=\|x\|_{\infty}+\left\|x^{\prime}\right\|_{\infty}, K=\{x \in E: x(t) \geqslant 0\}$. This cone is non-normal. Consider, for example, $x_{n}(t)=\frac{t^{n}}{n}$ and $y_{n}(t)=\frac{1}{n}$. Then $\theta \preceq x_{n} \preceq y_{n}$, and $\lim _{n \rightarrow \infty} y_{n}=\theta$, but $\left\|x_{n}\right\|=\max _{t \in[0,1]}\left|\frac{t^{n}}{n}\right|+\max _{t \in[0,1]}\left|t^{n-1}\right|=\frac{1}{n}+1>1$; hence $x_{n}$ does not converge to zero. It follows by $2^{\circ}$ that $K$ is a non-normal cone.

Let $X$ be a nonempty set and $(E, K)$ an ordered Banach space. A function $d: X \times X \rightarrow E$ is called a cone metric and $(X, d)$ is called a cone metric space if the following conditions hold:

(C1) $\theta \preceq d(x, y)$ for all $x, y \in X$ and $d(x, y)=\theta$ if and only if $x=y$;

(C2) $d(x, y)=d(y, x)$ for all $x, y \in X$;

(C3) $d(x, z) \preceq d(x, y)+d(y, z)$ for all $x, y, z \in X$.

Let $x \in X$ and $\left\{x_{n}\right\}$ be a sequence in $X$. Then it is said that:

(i) $\left\{x_{n}\right\}$ cone converges to $x$ if for every $c \in E$ with $\theta \ll c$ there exists a natural number $n_{0}$ such that $d\left(x_{n}, x\right) \ll c$ for all $n>n_{0}$; we denote it by $d-\lim _{n \rightarrow \infty} x_{n}=x$ or $x_{n} \stackrel{d}{\rightarrow} x$ as $n \rightarrow \infty$;

(ii) $\left\{x_{n}\right\}$ is a cone Cauchy sequence if for every $c \in E$ with $\theta \ll c$ there exists a natural number $n_{0}$ such that $d\left(x_{m}, x_{n}\right) \ll c$ for all $m, n>n_{0}$;

(iii) $(X, d)$ is cone complete if every tvs-Cauchy sequence is tvs-convergent in $X$.

Suppose now that $(X, d)$ is a cone metric space over a normal solid cone $K$ in a Banach space $E$ (with the normal constant satisfying $k \geqslant 1$ ). Then $D(x, y)=\|d(x, y)\|$ is a symmetric on the set $X$, that is, a mapping from $X \times X$ into $[0,+\infty)$ with the following properties:

(S1) $D(x, y) \geq 0$ and $D(x, y)=0$ if and only if $x=y$;

(S2) $D(x, y)=D(y, x)$.

It satisfies also:

(S3) $D(x, y) \leq k(D(x, z)+D(z, y))$.

The cone metric $d$ and the associated symmetric $D=\|d\|$ in $X$ generate two topologies: $t_{d}$ and $t_{D}$. Their bases of neighborhoods consist of the sets

$$
B_{c}(y)=\{x \in X: d(x, y) \ll c\} \text { and } B_{\varepsilon}(y)=\{x \in X: D(x, y)<\varepsilon\},
$$

where $y, c, \varepsilon$ are, respectively, a given point from $X$, a given interior point from $K$, and a given positive number (for details see [9]).

Theorem 2.1 [9] Let $(X, d)$ be a cone metric space with a normal solid cone $K$ and let $D$ be the associated symmetric. Then $t_{d}=t_{D}$.

In other words, the spaces $(X, d)$ and $(X, D)$ have the same collections of open, closed, bounded and compact sets, and also the same convergent and Cauchy sequences, and the same continuous functions. Also, the interior of the cone is the same in both equivalent norms. If the normal constant $k=1$, then the symmetric space $(X, D)$ is a metric space.

Thus we obtain the following principal remark: From Lemma 2.1 and Theorem 2.1 it is obvious that, in the investigation of cone metric spaces with normal solid cones, we can assume that the normal constant can be taken to be $k=1$. This follows from the fact that we can deal with the space $E$, equipped with the norm $\|\cdot\|_{1}$, which is equivalent to $\|\cdot\|$. 
Taking into account Lemma 2.1 and Theorem 2.1, it follows that results for normal solid cone metric spaces can be derived from the respective results for metric spaces. Namely, if $d$ is a cone metric on $X$ and the norm $\|\cdot\|$ is monotone on the cone, then the composition $\|\cdot\| \circ d=D$ is a usual metric on $X$. Clearly, non-expansive and contractive (with respect to $d$ ) self-mappings of $X$ are also non-expansive and contractive (respectively) with respect to the metric $\|\cdot\| \circ d=D$.

\section{Main results}

Let $(E,\|\cdot\|)$ be a Banach space with a cone $K \subset E$. Consider the set $B(K)$ of bounded linear operators $A$ on $E$ that are positive-definite, i.e., $A(K) \subset K$ holds. Moreover, let $B(K, 1)$ denote the subset of $B(K)$ containing operators $A$ having the norm $\|A\| \leqslant 1$. X. Zhang in [13], called a mapping $f: X \rightarrow X$ generalized operator quasicontractive on a cone metric space $(X, d)$ (over $K$ ) if there exists $\lambda \in[0,1)$ such that for all $x, y \in X$ there exist $a, b, c, d, e \geqslant 0$ satisfying $a+b+c+d+e \leqslant 1$, and $A, B, C, D, E \in B(K, 1)$ such that the following inequality holds

$$
d(f x, f y) \preceq \lambda(a A d(x, y)+b B d(x, f x)+c C d(y, f y)+d D d(x, f y)+e E d(y, f x)) .
$$

Note that the constants $a, b, c, d, e$, as well as the operators $A, B, C, D, E$ depend on the points $x, y$, but we will not write $a_{x, y}, \ldots$ in order to avoid cumbersome notation. The following example is inspired by [13, Example 1].

Example 3.1 Let $E=\mathbb{R}^{2}$ and let $K=\left\{(x, y)^{\top} \in \mathbb{R}^{2}: x \geq 0, y \geq 0\right\}$, which is a normal cone with $k=1$. Let $\mathscr{X}=\{a, b, c\}$ and define a cone metric $d$ on $\mathscr{X}$ by $d(x, x)=(0,0)^{\top}$ for $x \in \mathscr{X}, d(a, b)=d(a, c)=(1,1)^{\top}, d(b, c)=(0,1.1)^{\top}$ and $d(x, y)=d(y, x)$ for $x, y \in \mathscr{X}$. It is easy to see that $(\mathscr{X}, d)$ is a cone metric space.

Let $f: \mathscr{X} \rightarrow \mathscr{X}$ be defined by $f a=b, f b=f c=c$. Take $A=\left[\begin{array}{cc}0 & 0 \\ 0.6 & 0.6\end{array}\right] \in B(K, 1)$ and $\lambda=\frac{11}{12}$. Then it is easy to check that the inequality $d(f x, f y) \preceq \lambda A d(x, y)$ is fulfilled for all $x, y \in \mathscr{X}$. Hence, $f$ is a generalized operator quasicontractive mapping on $\mathscr{X}$.

X. Zhang proved the following

Theorem 3.1 [13] Let $(X, d)$ be a complete cone metric space over a normal cone $K$ with normal constant $k$. Let $f: X \rightarrow X$ be a generalized operator quasicontractive mapping with contractive constant $\lambda$. If one of the following conditions hold:

1. $f$ is continuous;

2. $\lambda k<1$;

3. there exists $x_{0} \in X$ such that

$$
\left\{A(x, y), B(x, y), C(x, y), D(x, y), E(x, y): x, y \in \mathscr{O}\left(x_{0}, \infty\right)\right\}
$$


is a compact set (here, $A(x, y), B(x, y), C(x, y), D(x, y), E(x, y)$ are operators $A, B, C, D, E$ from 3 and $\mathscr{O}\left(x_{0}, \infty\right)$ is the orbit of $f$ at the point $\left.x_{0}\right)$,

then $f$ has a unique fixed point $x^{*} \in X$. Moreover, in the cases (1) and (2), any Picard sequence $\left\{f^{n} x\right\}$ converges to $x^{*}$; in the case (3), the Picard sequence $\left\{f^{n} x_{0}\right\}$ converges to $x^{*}$.

We shall prove that the previous result can be obtained without assuming any of the conditions (1)-(3).

Theorem 3.2 Let $(X, d)$ be a complete cone metric space over a normal cone $K$ and let $f: X \rightarrow X$ be a generalized operator quasicontractive mapping. Then $f$ has a unique fixed point $x^{*} \in X$ and any Picard sequence $\left\{f^{n} x\right\}, x \in X$ converges to $x^{*}$.

Proof. According to Lemma $2.1,3^{\circ} \Leftrightarrow 4^{\circ}$, there exists a norm on $E$ (denoted by $\|\cdot\|$ ), equivalent to the original one, which is monotone, i.e., the implication 2 holds. As in Theorem 2.1, denote $D(x, y)=\|d(x, y)\|$. Then $D$ is a standard metric on $X$, and $(X, D)$ is a complete metric space. The inequality 3 implies that

$$
\begin{aligned}
& D(f x, f y) \leq\|d(f x, f y)\| \\
& \leq \lambda\|a A d(x, y)+b B d(x, f x)+c C d(y, f y)+d D d(x, f y)+e E d(y, f x)\| \\
& \leq \lambda(a\|A\|\|d(x, y)\|+b\|B\|\|d(x, f x)\|+c\|C\|\|d(y, f y)\| \\
&+d\|D\|\|d(x, f y)\|+e\|E\|\|d(y, f x)\|) \\
& \leq \lambda(a+b+c+d+e) \max \{\|d(x, y)\|,\|d(x, f x)\|,\|d(y, f y)\|, \\
&\|d(x, f y)\|, \| d(y, f x \|\} \\
& \leq \lambda \max \{\|d(x, y)\|,\|d(x, f x)\|,\|d(y, f y)\|,\|d(x, f y)\|, \| d(y, f x)\} \\
&= \lambda \max \{D(x, y), D(x, f x), D(y, f y), D(x, f y), D(y, f x)\}
\end{aligned}
$$

holds for all $x, y \in X$. In other words, $f$ is a Ćirić-type quasicontraction ([1]) in a complete metric space $(X, D)$. Hence, $f$ has a unique fixed point $x^{*} \in X$, being the limit of an arbitrary Picard sequence $\left\{f^{n} x\right\}$.

Remark 3.1 Since the mapping $f$ in Example 3.1 fulfills all the conditions of Theorem 3.2, it follows that $f$ has a unique fixed point (which is $x^{*}=c$ ). As was shown in a similar situation in [13, Example 1], no scalar cone-quasicontractive condition is satisfied in this case. However, the statement from [13, Remark 1] does not hold, since Theorem 3.2 and its proof show that the result can still be obtained by reducing it to the standard metric arguments. 


\section{References}

[1] Lj.B. Ćirić, A generalization of Banach's contraction principle, Proc. Amer. Math. Soc. 45 (1974), 267-273.

[2] K. Deimling, Nonlinear Functional Analysis, Springer-Verlag, 1985.

[3] D. Djukić, Lj. Paunović and S. Radenović,Convergence of iterates with eror of uniformly quasi-Lipschitzian mappings in cone metric spaces, Kragujevac J. Math., vol. 35, Number 3(2011), 399-410.

[4] Z. M. Fadail, A. G. B Ahmad and Ljiljana Paunović,New fixed point results of single-valued mapping for c-distance in cone metric spaces, Abstract and Applied Analysis, Article ID 639713 (2012) 12 pages.

[5] M. Filipović, Lj. Paunović, S. Radenović and M. Rajović, Remarks on Cone Metric Spaces and Fixed Point Theorems of T-Kannan Contractive Mappings, Math. And Computer Modeling, vol. 54, Issue 5-6,(2011), 1467-1472.

[6] L.G. Huang, X. Zhang, Cone metric spaces and fixed point theorems of contractive mappings, J. Math. Anal. Appl. 323 (2007), 1468-1476.

[7] S. Janković, Z. Kadelburg, S. Radenović, On cone metric spaces: A survey, Nonlinear Anal. TM\&A 74 (2011), 2591-2601.

[8] M. G. Krein, M. A. Rutman, Linear operators leaving invariant a cone in a Banach spaces, Uspekhi Math. Nauk (N.S.), 3, 1 (1948), 3-95.

[9] S. Radenović, Z. Kadelburg, Quasi-contractions on symmetric and cone symmetric spaces, Banach J. Math. Anal. 5, 1 (2011), 38-50.

[10] S. Radojević, Lj. Paunović and S. Radenović, Abstract metric spaces and Hardy-Rogers-type theorems, Appl. Math. Lett. 24(4) (2011), 553-558.

[11] J. S. Vandergraft, Newton's method for convex operators in partially ordered spaces, SIAM J. Numerical Anal. 4, 3 (1967), 406-432.

[12] P.P. Zabrě̌ko, K-metric and K-normed spaces: survey, Collectanea Math. 48, 4-6 (1997), 825-859.

[13] X. Zhang, Fixed point theorem for generalized operator quasi-contractive mappings in cone metric spaces, Afrika Mat., DOI 10.1007/s13370-012-0105-7. 